Bull. Korean Math. Soc. 51 (2014), No. 5, pp. 1425-1432

http://dx.doi.org/10.4134/BKMS.2014.51.5.1425

\title{
A NOTE ON RECURRENCE FORMULA FOR VALUES OF THE EULER ZETA FUNCTIONS $\zeta_{E}(2 n)$ AT POSITIVE INTEGERS
}

\author{
Hui Young Lee and Cheon Seoung Ryoo
}

\begin{abstract}
The Euler zeta function is defined by $\zeta_{E}(s)=\sum_{n=1}^{\infty} \frac{(-1)^{n-1}}{n^{s}}$. The purpose of this paper is to find formulas of the Euler zeta function's values. In this paper, for $s \in \mathbb{N}$ we find the recurrence formula of $\zeta_{E}(2 s)$ using the Fourier series. Also we find the recurrence formula of $\sum_{n=1}^{\infty} \frac{(-1)^{n-1}}{(2 n-1)^{2 s-1}}$, where $s \geq 2(\in \mathbb{N})$.
\end{abstract}

\section{Introduction}

The Euler zeta function is defined by $\zeta_{E}(s)=\sum_{n=1}^{\infty} \frac{(-1)^{n}}{n^{s}}($ see $[3,4])$. In this paper we investigate the recurrence formula of the Euler zeta function for $s=2 n$ with Fourier series. By this result we can find $\zeta_{E}(2 n)$ for all $n \in \mathbb{N}$.

For $s \in \mathbb{C}$, the Riemann zeta function or the Euler-Riemann zeta function, $\zeta(s)$ is defined by

$$
\zeta(s)=\sum_{n=1}^{\infty} \frac{1}{n^{s}} \quad(s \in \mathbb{C}), \quad(\text { see }[5,6])
$$

which converges when the real part of $s$ is greater than 1. R. Apéry proved that the number $\zeta(3)$ is irrational. But it is still an open problem to prove irrationality of $\zeta(2 k+1)$ for the long time.

As well known special values, for any positive even number $2 n$,

$$
\zeta(2 n)=(-1)^{n+1} \frac{B_{2 n}(2 \pi)^{2 n}}{2(2 n) !}, \quad(\text { see }[1])
$$

where $B_{2 n}$ are Bernoulli numbers. For negative integers, one has

$$
\zeta(-n)=-\frac{B_{n+1}}{n+1}
$$

for $n \geq 1$.

Received April 1, 2013; Revised November 21, 2013.

2010 Mathematics Subject Classification. Primary 42B05, 11B68, 11S40, 11S80.

Key words and phrases. zeta function, Euler zeta function, Fourier series.

This work was financially supported by Hannam University. 
The constants $E_{n}$ in the Taylor series expansion

$$
F(t)=\frac{2}{e^{t}+1}=\sum_{n=0}^{\infty} E_{n} \frac{t^{n}}{n !} \quad \text { for }|t|<\pi \quad(\text { see }[3,4,6,8]),
$$

are well known as the Euler number. The Euler polynomials are also defined by

$$
\frac{2}{e^{t}+1} e^{x t}=e^{E(x) t}=\sum_{n=0}^{\infty} E_{n}(x) \frac{t^{n}}{n !}, \quad|t|<\pi
$$

with the usual convention about replacing $E(x)^{n}$ by $E_{n}(x)$ (see $[2,3,4,5,6$, $7,8])$.

The recurrence formula of the Euler numbers is

$$
(E+1)^{n}+E_{n}= \begin{cases}2 & \text { if } n=0 \\ 0 & \text { if } n \geq 0 .\end{cases}
$$

In $[3,4]$, T. Kim find the relation of the Euler zeta function and the Euler numbers. From this result, one can obtain values of the Euler zeta function. But in our work, one has the recurrence formula of the Euler zeta function itself and as a result one has values of the Euler zeta function without Euler numbers in generally. Throughout this paper ${ }_{n} P_{r}$ denotes the permutation, where ${ }_{n} P_{r}=n(n-1)(n-2) \cdots(n-r+1)$.

\section{Fourier series}

Definition 1 (Fourier Series). Let $f(x)$ be function on $(-p, p)$ with

$$
f(x)=\frac{a_{0}}{2}+\sum_{k=1}^{\infty}\left(a_{k} \cos \frac{k \pi x}{p}+b_{k} \sin \frac{k \pi x}{p}\right) \quad(\text { see }[9]) .
$$

Then it is called the Fourier series and coefficients are $a_{0}=\frac{1}{p} \int_{-p}^{p} f(x) d x$, $a_{n}=\frac{1}{p} \int_{-p}^{p} f(x) \cos \frac{n \pi x}{p} d x, b_{n}=\frac{1}{p} \int_{-p}^{p} f(x) \sin \frac{n \pi x}{p} d x$.

Note that the Fourier series of $f$ on $(-p, p)$ converges to $f(x)$ at points of continuity and converges to $\frac{f(x+)+f(x-)}{2}$ at the point of discontinuities, where $f(x+)$ is the limit of right side at $x, f(x-)$ is the limit of left side at $x$.

Remark. From $p$-test we know that

$$
\sum_{n=1}^{\infty} \frac{1}{n^{p}}= \begin{cases}\text { absolutely convergence } & \text { if } p>1 \\ \infty & \text { if } p \leq 1\end{cases}
$$

If $p=2$, then $\sum_{n=1}^{\infty} \frac{1}{n^{2}}=\zeta(2)=\frac{\pi^{2}}{6}$. Also

$$
\sum_{n=1}^{\infty} \frac{1}{n^{2}}=\sum_{n=1}^{\infty} \frac{1}{(2 n)^{2}}+\sum_{n=1}^{\infty} \frac{1}{(2 n-1)^{2}}=\frac{1}{4} \zeta(2)+\sum_{n=1}^{\infty} \frac{1}{(2 n-1)^{2}}
$$


Hence, we get the following:

$$
\sum_{n=1}^{\infty} \frac{1}{(2 n-1)^{2}}=\frac{3}{4} \sum_{n=1}^{\infty} \frac{1}{n^{2}}=\frac{3}{4} \zeta(2)=\frac{\pi^{2}}{8} .
$$

Let us consider $f(x)$ on $(-p, p)$. If one takes that $f$ is an even function on $(-p, p)$, then one has coefficients as below:

$$
\begin{aligned}
& a_{0}=\frac{1}{p} \int_{-p}^{p} f(x) d x=\frac{2}{p} \int_{0}^{p} f(x) d x \\
& a_{n}=\frac{1}{p} \int_{-p}^{p} f(x) \cos \frac{n \pi x}{p} d x=\frac{2}{p} \int_{0}^{p} f(x) \cos \frac{n \pi x}{p} d x \\
& b_{n}=\frac{1}{p} \int_{-p}^{p} f(x) \sin \frac{n \pi x}{p} d x=0 .
\end{aligned}
$$

Then the Fourier series of $f$ on $(-p, p)$ is given by

$$
f(x)=\frac{a_{0}}{2}+\sum_{n=1}^{\infty} a_{n} \cos \frac{n \pi x}{p},
$$

where $a_{0}=\frac{2}{p} \int_{0}^{p} f(x) d x, a_{n}=\frac{2}{p} \int_{0}^{p} f(x) \cos \frac{n \pi x}{p} d x$. Then it is called the cosine series (see [9]).

Let $f$ be an odd function on $(-p, p)$. Then coefficients are as below;

$$
\begin{aligned}
& a_{0}=\frac{1}{p} \int_{-p}^{p} f(x) d x=0 \\
& a_{n}=\frac{1}{p} \int_{-p}^{p} f(x) \cos \frac{n \pi x}{p} d x=0 \\
& b_{n}=\frac{1}{p} \int_{-p}^{p} f(x) \sin \frac{n \pi x}{p} d x=\frac{2}{p} \int_{0}^{p} f(x) \sin \frac{n \pi x}{p} d x .
\end{aligned}
$$

Then the Fourier series of $f$ on $(-p, p)$ is given by

$$
f(x)=\sum_{n=1}^{\infty} b_{n} \sin \frac{n \pi x}{p}
$$

where $b_{n}=\frac{2}{p} \int_{0}^{p} f(x) \sin \frac{n \pi x}{p} d x$. Then it is called the sine series (see [9]).

Example 2. The sine series of Square wave function is given by

$$
f(x)= \begin{cases}-1 & \text { if }-\pi<x<0 \\ 1 & \text { if } 0 \leq x<\pi\end{cases}
$$

Above the Square wave function is an odd function and one has the sine series as below:

$$
f(x)=\sum_{n=1}^{\infty} b_{n} \sin n x,
$$

where $b_{n}=\frac{2}{\pi} \int_{0}^{\pi} 1 \cdot \sin n x d x=\frac{2}{\pi}\left(\frac{1-(-1)^{n}}{n}\right)$. 
Hence

$$
f(x)=\frac{4}{\pi} \sum_{n=1}^{\infty} \frac{1}{2 n-1} \sin (2 n-1) x .
$$

Thus, we note that

$$
1=f\left(\frac{\pi}{2}\right)=\frac{4}{\pi} \sum_{n=1}^{\infty} \frac{1}{2 n-1} \sin \frac{2 n-1}{2} \pi=\frac{4}{\pi} \sum_{n=1}^{\infty} \frac{(-1)^{n-1}}{2 n-1} .
$$

Therefore one has as below:

$$
\sum_{n=1}^{\infty} \frac{(-1)^{n-1}}{2 n-1}=\frac{\pi}{4}
$$

It is used as the initial value for $\sum_{n=1}^{\infty} \frac{(-1)^{n-1}}{(2 n-1)^{2 s-1}}$ in Section 4 .

\section{Euler- $\zeta$-function}

The Euler- $\zeta$-function is well known as below:

$$
\zeta_{E}(s)=\sum_{n=1}^{\infty} \frac{(-1)^{n-1}}{n^{s}}, \quad s \in \mathbb{C} \quad(\text { see }[4]) .
$$

Example 3. Let $f(x)=x^{2},-2<x<2$ which is an even function. Then

$$
f(x)=\frac{a_{0}}{2}+\sum_{n=1}^{\infty} a_{n} \cos \frac{n \pi x}{2},
$$

where $a_{0}=\frac{2}{2} \int_{0}^{2} x^{2} d x=\frac{8}{3}, a_{n}=\frac{2}{2} \int_{0}^{2} x^{2} \cos \frac{n \pi x}{2} d x=\frac{16(-1)^{n}}{(n \pi)^{2}}$.

Hence one has the following cosine series:

$$
f(x)=\frac{4}{3}+\frac{16}{\pi^{2}} \sum_{n=1}^{\infty} \frac{(-1)^{n}}{n^{2}} \cos \frac{n \pi x}{2}, \quad-2<x<2 .
$$

Taking $x=2$ in $f(x)$, we get $\zeta(2)=\frac{\pi^{2}}{6}$. Taking $x=1$ in $f(x)$, we get $\zeta_{E}(2)=\frac{\pi^{2}}{12}$, which is used as the initial value for $\zeta_{E}(2 n)$ in Section 4 .

Let $f(x)=x^{3},-2<x<2$. Then the sin series of $f(x)$ on $(-2,2)$ is given by

$$
f(x)=\sum_{n=1}^{\infty} b_{n} \sin \frac{n \pi x}{2}
$$

where $b_{n}=\frac{2}{2} \int_{0}^{2} x^{3} \sin \frac{n \pi x}{2} d x=-\frac{(-1)^{n} 2^{4}}{n \pi}+\frac{(-1)^{n} 2^{5} \times 3}{n^{3} \pi^{3}}$. Then, if we take $x=1$ in (7), we get easily the following:

$$
\sum_{n=1}^{\infty} \frac{(-1)^{n-1}}{(2 n-1)^{3}}=\frac{\pi^{3}}{2^{5}}
$$


Let $f(x)=x^{4},-2<x<2$. Then the cosine series of $f(x)$ on $(-2,2)$ is given by

$$
f(x)=\frac{a_{0}}{2}+\sum_{n=1}^{\infty} a_{n} \cos \frac{n \pi x}{2},
$$

where $a_{0}=\frac{2^{5}}{5}, a_{n}=\frac{(-1)^{n} 2^{7}}{n^{2} \pi^{2}}-\frac{(-1)^{n} 2^{8} \times 3}{n^{4} \pi^{4}}$. Then, if we take $x=1$ in (8), we get easily the following:

$$
\zeta_{E}(4)=\sum_{n=1}^{\infty} \frac{(-1)^{n-1}}{n^{4}}=\frac{7 \pi^{4}}{2^{4} \times 3^{2} \times 5} .
$$

4. Recurrence formulas of the Euler zeta function $\zeta_{E}(2 s)$ for $s \in \mathbb{N}$ and $\sum_{n=1}^{\infty} \frac{(-1)^{n-1}}{(2 n-1)^{2 s-1}}$ for $s \in \mathbb{N}$

To generalize this process, we consider two functions $f(x)=x^{2 m}, f(x)=$ $x^{2 m-1}$, where $-2<x<2$. Firstly, let $f(x)=x^{2 m}$ on $-2<x<2$. Since $f(x)$ is an even function, we apply the cosine series for $f(x)$ is given by

$$
f(x)=\frac{a_{0}}{2}+\sum_{n=1}^{\infty} a_{n} \cos \frac{n \pi x}{2}, \quad-2<x<2,
$$

where $a_{0}=\frac{2^{2 m+1}}{2 m+1}, a_{n}=\sum_{k=1}^{m}(-1)^{k+1}{ }_{2 m} P_{2 k-1} 2^{2 m-2 k+1} \frac{2^{2 k}}{n^{2 k} \pi^{2 k}} \cos n \pi$.

From $(9)$, for $s \geq 2(\in \mathbb{N})$ we take the following two formulas:

$$
\begin{gathered}
\sum_{k=1}^{s}(-1)^{k}{ }_{2 s} P_{2 k-1} \frac{1}{2^{2 k} \pi^{2 k}} \zeta_{E}(2 k)=\frac{2 s+1-2^{2 s}}{(2 s+1)\left(2^{2 s+1}\right)} \\
\sum_{k=1}^{s-1}(-1)^{k}{ }_{2 s-2} P_{2 k-1} \frac{1}{2^{2 k} \pi^{2 k}} \zeta_{E}(2 k)=\frac{2 s-1-2^{2(s-1)}}{(2 s-1)\left(2^{2 s-1}\right)} .
\end{gathered}
$$

From (10) and (11) we get the formula as below:

$$
\begin{aligned}
\zeta_{E}(2 s)= & \frac{(2 \pi)^{2 s}}{(-1)^{s}{ }_{2 s} P_{2 s-1}}\left\{\frac{1}{2^{2 s+1}} \frac{2^{2 s+1}-12 s^{2}+3}{(2 s-1)(2 s+1)}\right. \\
& \left.-\sum_{k=1}^{s-1}(-1)^{k} \frac{1}{(2 \pi)^{2 k}} \zeta_{E}(2 k)\left({ }_{2 s} P_{2 k-1}-{ }_{2 s-2} P_{2 k-1}\right)\right\} .
\end{aligned}
$$

Therefore from (12) one has the following theorem.

Theorem 4. For $s \geq 2(\in \mathbb{N})$ and $\zeta_{E}(2)=\frac{\pi^{2}}{12}$, we get as below:

$$
\begin{aligned}
\zeta_{E}(2 s)= & \frac{(2 \pi)^{2 s}}{(-1)^{s}{ }_{2 s} P_{2 s-1}}\left\{\frac{1}{2^{2 s+1}} \frac{2^{2 s+1}-12 s^{2}+3}{(2 s-1)(2 s+1)}\right. \\
& \left.-\sum_{k=1}^{s-1}(-1)^{k} \frac{1}{(2 \pi)^{2 k}} \zeta_{E}(2 k)\left({ }_{2 s} P_{2 k-1}-{ }_{2 s-2} P_{2 k-1}\right)\right\},
\end{aligned}
$$


where ${ }_{n} P_{r}$ is the permutation.

Note that T. Kim find the Euler zeta function with using the second kind Euler numbers as follows:

$$
\zeta_{E}(2 n)=\frac{(-1)^{n} \pi^{2 n}\left(2-4^{n}\right)}{2(2 n-1) !\left(1-4^{n}\right)} E_{2 n-1} \quad(\text { see [4]). }
$$

Secondly, let $f(x)=x^{2 m-1}$ on $-2<x<2, m \geq 1$. The sin series of $f(x)$ on $-2<x<2$ is given by

$$
f(x)=\sum_{n=1}^{\infty} b_{n} \sin \frac{n \pi x}{2},
$$

where $b_{n}=\int_{0}^{2} x^{2 m-1} \sin \frac{n \pi x}{2} d x$.

By simple calculations we get the coefficient $b_{n}$ as below:

$$
b_{n}=(-1)^{n} 2^{2 m} \sum_{k=1}^{m}(-1)^{k}{ }_{2 m-1} P_{2(k-1)}\left(\frac{1}{n \pi}\right)^{2 k-1} \text {. }
$$

So we apply the sin series to above (14) and we take $x=1$. Then one has the following:

$$
\begin{aligned}
1 & =\sum_{n=1}^{\infty}(-1)^{n} 2^{2 m} \sum_{k=1}^{m}(-1)^{k}{ }_{2 m-1} P_{2(k-1)}\left(\frac{1}{n \pi}\right)^{2 k-1} \sin \frac{n \pi}{2} \\
& =\sum_{n=1}^{\infty}(-1)^{2 n-1} 2^{2 m} \sum_{k=1}^{m}(-1)^{k}{ }_{2 m-1} P_{2(k-1)}\left(\frac{1}{(2 n-1) \pi}\right)^{2 k-1}(-1)^{n-1} \\
& =2^{2 m} \sum_{k=1}^{m}(-1)^{k-1}{ }_{2 m-1} P_{2(k-1)} \frac{1}{\pi^{2 k-1}} \sum_{n=1}^{\infty} \frac{(-1)^{n-1}}{(2 n-1)^{2 k-1}} .
\end{aligned}
$$

From (15), we get following two relations:

$$
\begin{gathered}
\frac{1}{2^{2 s}}=\sum_{k=1}^{s}(-1)^{k-1}{ }_{2 s-1} P_{2(k-1)} \frac{1}{\pi^{2 k-1}} \sum_{n=1}^{\infty} \frac{(-1)^{n-1}}{(2 n-1)^{2 k-1}}, \\
\frac{1}{2^{2(s-1)}}=\sum_{k=1}^{s-1}(-1)^{k-1}{ }_{2 s-3} P_{2(k-1)} \frac{1}{\pi^{2 k-1}} \sum_{n=1}^{\infty} \frac{(-1)^{n-1}}{(2 n-1)^{2 k-1}} .
\end{gathered}
$$

From (16)-(17), one has the following formula:

$$
\begin{aligned}
& \sum_{n=1}^{\infty} \frac{(-1)^{n-1}}{(2 n-1)^{2 s-1}} \\
= & \frac{(-1)^{s-1} \pi^{2 s-1}}{2 s-1} P_{2(s-1)}\left[-\frac{3}{4^{s}}\right. \\
& \left.\quad+\sum_{k=1}^{s-1}(-1)^{k} \frac{1}{\pi^{2 k-1}}\left(\sum_{n=1}^{\infty} \frac{(-1)^{n-1}}{(2 n-1)^{2 k-1}}\left(2 s-1 P_{2(k-1)}-{ }_{2 s-3} P_{2(k-1)}\right)\right)\right] .
\end{aligned}
$$


Theorem 5. For $s \geq 2(\in \mathbb{N})$ and $\sum_{n=1}^{\infty} \frac{(-1)^{n-1}}{2 n-1}=\frac{\pi}{4}$, one has as below:

$$
\begin{aligned}
& \sum_{n=1}^{\infty} \frac{(-1)^{n-1}}{(2 n-1)^{2 s-1}} \\
= & \frac{(-1)^{s-1} \pi^{2 s-1}}{2 s-1} P_{2(s-1)}\left[-\frac{3}{4^{s}}\right. \\
& \left.\quad+\sum_{k=1}^{s-1}(-1)^{k} \frac{1}{\pi^{2 k-1}}\left(\sum_{n=1}^{\infty} \frac{(-1)^{n-1}}{(2 n-1)^{2 k-1}}\left(2 s-1 P_{2(k-1)}-{ }_{2 s-3} P_{2(k-1)}\right)\right)\right],
\end{aligned}
$$

where ${ }_{n} P_{r}$ is the permutation.

Note that T. Kim find the relationship between $\sum_{k=0}^{\infty} \frac{(-1)^{k}}{(2 k+1)^{(2 n+1)}}$ and the second kind Euler polynomials as follows:

$$
\sum_{k=0}^{\infty} \frac{(-1)^{k}}{(2 k+1)^{(2 n+1)}}=(-1)^{n} \frac{E_{2 n}\left(\frac{1}{2}\right)}{(2 n) ! 4} \pi^{2 n+1} \quad \text { (see [4]). }
$$

Acknowledgements. We thank Prof. Kim Tae-kyun of Kwang-woon University whose lectures on theories and careful comments on this paper are greatly appreciated.

\section{References}

[1] R. Ayoub, Euler and zeta function, Amer. Math. Monthly 81 (1974), 1067-1086.

[2] T. Kim, On the analogs of Euler numbers and polynomials associated with $p$-adic qintegral on $\mathbb{Z}_{p}$ at $q=-1$, J. Math. Anal. Appl. 331 (2007), no. 2, 779-792.

[3] _ Euler numbers and polynomials associated with zeta functions, Abstr. Appl. Anal. 2008 (2008), Article ID 581582, 11 pages.

[4] T. Kim, J. Choi, and Y. H. Kim, A note on values of Euler zeta functions at positive integers, Adv. Stud. Contemp. Math. 22 (2012), no. 1, 27-34.

[5] D. S. Kim and T. Kim, Euler basis, identities, and their applications, Int. J. Math. Math. Sci. 2012 (2012), Article ID 343981, 15 pages.

[6] D. S. Kim, T. Kim, W. J. Kim, and D. V. Dolgy, A note on Eulerian polynomials, Abstr. Appl. Anal. 2012 (2012), Article ID 269640, 10 pages.

[7] H. Ozden, Y. Simsek, S.-H. Rim, and I. N. Cangul, A note on p-adic q-Euler measure, Adv. Stud. Contemp. Math. 14 (2007), no. 2, 233-239.

[8] S. H. Rim and T. Kim, A note on q-Euler numbers associated with the basic q-zeta function, Appl. Math. Lett. 20 (2007), no. 4, 366-369.

[9] D. G. Zill and W. S. Wright, Advanced Engineering Mathematics. 4th, Textbooks, 2009.

Hui Young Lee

Department of Mathematics

HANNAM UNIVERSITY

DAEJEON 306-791, KoreA

E-mail address: normaliz@hnu.kr 
Cheon Seoung Ryoo

Department of Mathematics

HANNAM UNIVERSITY

DAEJEON 306-791, KoreA

E-mail address: ryoocs@hnu.kr 